MS16-P23

\section{1:3 A-site ordering in perovskite structures: group theoretical analysis}

Mikhail Talanov ${ }^{1}$

1. Southern Federal University, Rostov-on-Don, Russia

email: tmikle-man@mail.ru

The quadruple perovskites $\mathrm{AA}_{3}{ }_{3} \mathrm{~B}_{4} \mathrm{O}_{12}$ are characterized by an extremely wide variety of intriguing physical properties, which make them demanded for various applications [1,2]. Using group-theoretic analysis, we found all possible 1:3 A-site ordered low-symmetry phases, which can be formed from a parent $\mathrm{Pm}-3 \mathrm{~m}$ perovskite structure (archetype) as a result of real or hypothetical (virtual) phase transitions due to different structural mechanisms (ordering and displacement of atoms, anion octahedral tilts). For each of the low-symmetry phases, the complete condensate of order parameters (proper and improper order parameters), the calculated structure, including the space group, the primitive cell multiplication, the distribution of atoms over the Wyckoff positions and the structural formula were determined. All phases were systematized according to the types of structural mechanisms responsible for their formation, and the group-subgroup relations between the phases were investigated. Special attention is paid to the structural mechanisms for the low-symmetry phase's formation of the compounds known from experimental data, such as: $\mathrm{CaCu}-$ ${ }_{3} \mathrm{Ti}_{4} \mathrm{O}_{12}, \mathrm{CaCu}_{3} \mathrm{Ga}_{2} \mathrm{Sn}_{2} \mathrm{O}_{12}, \mathrm{CaMn}_{3} \mathrm{Mn}_{4} \mathrm{O}_{12}, \mathrm{Ce}_{1 / 2} \mathrm{Cu}_{3} \mathrm{Ti}_{4} \mathrm{O}_{12}$, $\mathrm{LaMn}_{3} \mathrm{Mn}_{4} \mathrm{O}_{12}$ and others. For the first time, the variability in the choice of the proper order parameters, which generate 1: 3 A-site ordered low-symmetry phases, highlights the fundamental significance of the complete condensate of the order parameters in the description of structural phase transitions. The constructed map of the 1:3 A-site ordered quadruple perovskites can be used to identify new phases and interpret experimental results, determine the structural mechanisms responsible for the formation of low-symmetry phases as well as to understand the structural genesis of the perovskite-like phases. The obtained non-model group-theoretical results in combination with crystal-chemical data and first principles calculations can be a starting point for the design of new functional materials with a perovskite structure.

The reported study was funded by RFBR - research projects no.16-32-60025 mol_a_dk.

References:

[1] Shimakawa, Y. (2008). Inorg. Chem., 47, 8562-8570.

[2] Belik, A.A.. (2018). Dalt. Trans., 47, 3209-3217.

Keywords: quadruple perovskites, group-theoretical analysis, order parameter
MS16-P24

\section{Crystallographic studies of $\mathrm{NaZr}_{2}\left(\mathrm{PO}_{4}\right)_{3}$ phosphates at high temperatures}

Albina Orlova ${ }^{1}$, Sergei Khainakov², Aleksey Alexandrov ${ }^{1}$, Santiago

Garcia-Granda $^{2}$, Dmitrii Savinykh ${ }^{1}$

1. Lobachevsky State University of Nizhni Novgorod, Nizhni

Novgorod, Russia

2. University of Oviedo, Oviedo, Spain

email: albina.orlova@inbox.ru

Crystal-chemical principles have been used in this paper for modeling of the compositions of $\mathrm{NaZr}_{2}\left(\mathrm{PO}_{4}\right)_{3}$ compounds and solid solutions with adjustable thermal expansion characteristics. Knowledge about materials on their base helps to solve many engineering problems associated with the combination of materials in different designs, including the required resistance to thermal stress.

$\mathrm{NaZr}_{2}\left(\mathrm{PO}_{4}\right)_{3}(\mathrm{NZP})$ is a convenient structure for these aims as it offers an opportunity to include a big variety of cations in different combinations with oxidation states from +1 to +5 , with different size and electronegativity into the composition of isostructural phases.

Well-known NZP materials have high melting temperatures, high hardness, thermomechanical, radiation, and chemical stability, ion conductivity, optical and magnetic properties, catalytic activity.

Although many of the possible formula compositions calculated in [1] are well studied, but those that have their thermal expansion characteristics determined are quite few.

In this paper new phosphates $\mathrm{Na}_{1+2 \mathrm{x}} \mathrm{Zr}_{2-\mathrm{x}} \mathrm{Co}_{\mathrm{x}}\left(\mathrm{PO}_{4}\right)_{3}$ and $\mathrm{Ca}_{0,5+\mathrm{x}} \mathrm{Zr}_{2-\mathrm{x}} \mathrm{Co}_{\mathrm{x}}\left(\mathrm{PO}_{4}\right)_{3} \quad(0 \leq \mathrm{x} \leq 0,5)$ in various concentration combinations of zirconium and cobalt in the structure framework and with respectively changing population density of interframe positions are obtained and characterized, their behavior during heating through high-temperature $\mathrm{XRD}$ is studied for $\mathrm{T}=25-700^{\circ} \mathrm{C}$.

Synthesis of powder samples was carried out using solid-phase processes $\left(\mathrm{T}_{\text {final }}=700^{\circ} \mathrm{C}\right)$. The forming of phases of a planed composition and structure were established by XRD and SEM methods. Lattice parameters of the obtained materials were determined and their dependencies on the composition and temperature were analyzed.

It was found that with the temperature rising, $a$ and $b$ parameters were decreased, while $c$ parameter was increased, which is typical for NZP structure. With cobalt growing in the phase composition, absolute values of thermal expansion linear coefficients were decreased: $\alpha_{a}$ from $-4,65 \cdot 10^{-6}$ to $-3,06 \cdot 10^{-6}{ }^{\circ} \mathrm{C}^{-1}, \alpha_{c}$ from $21,85 \cdot 10^{-6}$ to $19,49 \cdot 10^{-6}{ }^{\circ} \mathrm{C}^{-1}$ for Na-containing series, $\alpha_{a}$ from $-1,82 \cdot 10^{-6}$ to $-1,14 \cdot 10^{-6}{ }^{\circ} \mathrm{C}^{-1}$, $\alpha_{c}$ from $9,20 \cdot 10^{-6}$ to $9,10 \cdot 10^{-6}{ }^{\circ} \mathrm{C}^{-1}$ for Ca-containing series and anisotropy: $\Delta \alpha$ from $26,50 \cdot 10^{-6}$ to $21,55 \cdot 10^{-6}{ }^{\circ} \mathrm{C}^{-1}$ for $\mathrm{Na}$-containing series, from $11,02 \cdot 10^{-6}$ to $10,24 \cdot 10^{-6}{ }^{\circ} \mathrm{C}^{-1}$ for Ca-containing series.

Analysis of the obtained results shows that the phosphates studied belong to the class of medium-expanders ones.

The studied objects have blue color. This property is useful for heat-resistant inorganic dyes applied as a cover on the material, as well as for imparting a functional color to the material itself. 
The study has been performed with the financial support of the Russian Science Foundation Grant No. 16-13-10464.

References:

[1] Orlova A. I. (2002). Radiochemistry. 44, 423-445.

Keywords: NZP, thermal expansion

\section{VS16-P25}

Effect of crystallography on the calculation energies of equilibrium and enthalpies of formations at first principles calculations

\section{(D.F.T)}

Ferroudj abdelhak $^{1}$, Y. Djaballah ${ }^{1}$, Dj. Belbacha ${ }^{1}$

1. Laboratoire d'Etudes Physico-Chimique des Matériaux

(L.E.P.C.M) Batna 1 University, Batna, Algeria

email: ferroudjabdelhak@yahoo.fr

Equilibrium energies and enthalpies of formations are calculated based on structural crystallography. Elements and alloys having a large number of atoms are difficult to be compute by density functional theory (DFT): time computation, points of high symmetry.

We have calculated the structural properties, the formation enthalpies and total energies of elements $\mathrm{Si}, \mathrm{Ni}$ and $\mathrm{Au}-\mathrm{Re}$ binary compounds $(\mathrm{Re}=\mathrm{Y}, \mathrm{La}, \mathrm{Eu})$ using the first principles calculations based on the density functional theory (DFT) byVasp package.

Comparison of structural properties, formation enthalpies and total energies obtained using firstly the primitive unit cell and secondly the Conventional unit cell of the same phase present a good agreement between the two cases.

The benefits of crystallography allowed the abintio simulator to save computing time; by the exact determination of the atomic positions and symmetry space group of such a material.

Keywords: crystallography, first principles calculation ,enthalpies formation 